\title{
PROGRAMA DE ESTRATEGIAS PARA MEJORAR LOS LOGROS DE APRENDIZAJE EN LOS NIÑOS Y NIÑAS CON TRASTORNO DE DEFICIT DE ATENCIÓN E HIPERACTIVIDAD (TDAH)
}

\section{PROGRAM STRATEGIES TO IMPROVE LEARNING ACHIEVEMENT IN CHILDREN WITH ADHD}

\section{RESUMEN}

${ }^{1}$ Nazario Aguirre B ${ }^{a},{ }^{2}$ Edith Gavidia O ${ }^{\text {a }},{ }^{3}$ Edith Aguirre $\mathrm{G}^{\text {a }}{ }^{4}$, Yessika Aguirre $\mathrm{G}^{\mathrm{a}}$

La teoría de Piaget es interaccionista; es decir, el crecimiento de la inteligencia se encuentra sujeto a un mecanismo regulador, denominado "FACTOR DE EQUILIBRIO", el cual interacciona las causas del desarrollo de la inteligencia: la herencia, la maduración psicológica, el ambiente. El presente estudio asume una perspectiva teórica que redimensiona el concepto del diseño teórico y metodológico de la investigación.

Asimismo, se exponen la metodología y los resultados de un diagnóstico en diferentes aspectos, relacionados con la institución educativa materia de investigación, teniendo como objetivo proponer y aplicar el Programa de Estrategia, para mejorar los logros de aprendizaje de los niños y niñas con Trastorno de Déficit Atención e Hiperactividad.

El diseño es de tipo cuasi experimental y como muestra representativa se consideró a 10 estudiantes, siendo 5 del grupo experimental y los otros 5 el grupo control, se evaluó sus logros de aprendizaje antes y después del programa, diagnosticándose a través de la Escala de Conners 10 casos de estudiantes con Trastorno de Déficit Atención e Hiperactividad, cuyos resultados arrojaron que los niños del grupo experimental y control antes de aplicar el programa estaban catalogados en fase de inicio y después de concluido el programa están como logros destacados.

Palabras clave: Déficit de atención, logros de aprendizaje, programa de estrategias.

\section{ABSTRACT}

Piaget's theory is interactionist. The growing of intelligence is based upon a regulatory mechanism called "Factor of Equilibrium" which interacts with the causes of intelligence development: inheritance, psychological growth, and environment.

This study assumes a theoretical perspective that resizes the concept of both elements the theoretical and methodological design of this research. Likewise, it is exposed the methodology and results of a diagnosis that covers different aspects related to the edcuational institution that is the subject of this investigation. Furthermore, this study aims to propose and apply a program of strategies to improve learning achievements in children with Attention Deficit/Hyperactivity Disorder (ADHD).

The design of this research is quasi-experimental and considered 10 children as a representative sample distributed into two groups: 5 students as part of the experimental group and 5 students as part of the control group. The student's learning achievements were measured before and after the implementation of this program using the Conner's Scale.

Regarding the ADHD diagnosis, the results revealed that the learning achievements of the children in both groups, the experimental and the control group, were catalogued in the start-up phase; and by the completion of the program the same students were catalogued as outstanding achievements.

Keywords: attention deficit, learning achievements, program strategies

${ }^{1}$ Universidad Nacional de Jaén. Cajamarca. Perú.

${ }^{\text {a }}$ Lic. en Educación. 


\section{INTRODUCCIÓN}

El programa de estrategias metodológicas que proponemos se enmarca en un contexto cuyo objetivo es mejorar los logros de aprendizaje de los niños y niñas con Trastorno de Déficit Atención e Hiperactividad (TDAH), y desarrollar actitudes de convivencia pacífica en un ambiente de respeto donde prevalezca la participación democrática respetando las opiniones de los demás y consigo mismo, teniendo en cuenta que se trata de un problema tan antiguo y generalizado como la propia escuela tradicional. Es así como los primeros resultados obtenidos son alarmantes, ya que al realizar un sondeo de opinión sobre agresiones específicas, pareciera que a lo largo de su vida en la escuela y en la familia no han sido acogidos y apoyados para superar esta limitación.

En este sentido, el grupo de trabajo consideramos que la escuela debe manifestar un especial interés en mejorar los logros de aprendizaje de los niños y niñas con Trastorno de Déficit Atención e Hiperactividad (TDAH), poniendo como énfasis la educación en valores porque no podemos seguir permitiendo que alumnos y alumnas sean discriminados por no conocer su realidad personal y psicosocial.

Se caracterizó la situación problemática sobre el bajo nivel de desempeño del docente tutor; así como a los antecedentes de investigación que se han realizado sobre este tipo de problemas, tanto a nivel local, nacional e internacional. Los enfoques teóricos, tratados por entender y buscar estrategias de solución.

Se presentan las conclusiones a las que se arribaron, luego del análisis e interpretación de los resultados obtenidos después de la aplicación del pos-test; las referencias bibliográficas y otros medios consultados; así como los anexos que servirán para explicar y aclarar el proceso de investigación.
El aporte del trabajo de investigación radica en la propuesta de un programa de estrategias para mejorar los logros de aprendizaje en los niños y niñas con Trastorno de Déficit Atención e Hiperactividad de educación primaria de la institución educativa $\mathrm{N}^{\circ} 16001$ de la ciudad de Jaén en el año 2014, para ser aplicado a estudiantes con trastorno de déficit de atención e hiperactividad. (TDAH).

\section{MATERIAL Y MÉTODOS.}

La presente investigación realizada ha sido de tipo aplicativo. Según Sánchez \& Reyes (2006) este tipo de investigación permitió orientar y describir, explicar y predecir la realidad, teniendo en cuenta el programa de estrategias implementadas.

\section{Los métodos utilizados fueron:}

El método de análisis - síntesis. Para su fundamentación teórica, elaboración del programa, valoración de los resultados, procesamiento de la información de las diferentes fuentes bibliográficas y virtuales y caracterización del objeto de estudio.

El método sistémico. Para determinar la estructura del diseño del programa y establecer las relaciones necesarias que se producen entre sus componentes de y la formulación de los factores.

Análisis Documental: para identificar el contexto y organizar las referencias bibliográficas especializadas sobre: Manifestaciones del trastorno, trastorno de déficit de atención e hiperactividad. (TDAH), logros de aprendizaje, competencias del docente para afrontar el desafío de una educación inclusiva.

\section{Instrumentos:}

Los instrumentos para la recolección de datos para la variable independiente fueron una ficha de observación y para la variable dependiente un cuestionario. 
a. En la ficha de observación se consignó la planificación y ejecución de la adecuación curricular para atender a niños y niñas con necesidades educativas especiales. En cuanto a su estructura se tuvo en cuenta los indicadores e índices establecidos para las adecuaciones curriculares de acceso, adecuaciones a los elementos básicos del currículo y adecuaciones de contexto. Dicha valoración se hizo mediante pre y pos prueba aplicado a las unidades muéstrales.

b. Tabla de evaluación, Fueron validados mediante dos dimensiones (atención, hiperactividad, impulsividad) y 14 preguntas, las mismas que permitirán identificar los criterios de diagnóstico para los niños y niñas con TDAH, la cual se aplicó a los padres y madres de familia.

c. Escala de evaluación para el TDAH. (EDHA). Permitió identificar las dimensiones e indicadores del TDAH dentro del aula de clase, por lo cual será resuelto por los docentes a través de una escala valorativa del 0 al 3 .

d. Libreta de notas, se empleó para registrar las actividades más significativas realizadas en el proceso de la investigación.

e. Pre Test: Es un instrumento que permitió medir y evaluar las capacidades cognitivas de los estudiantes.

\section{RESULTADOS}

RESULTADOS SEGÚN EVALUACIÓN A PADRES DE FAMILIA SOBRE TDAH EN LOS NIÑOS Y NIÑAS DE LA INSTITUCIÓN EDUCATIVA N 16001 DE LA CIUDAD DE JAÉN EN EL AÑO 2014.

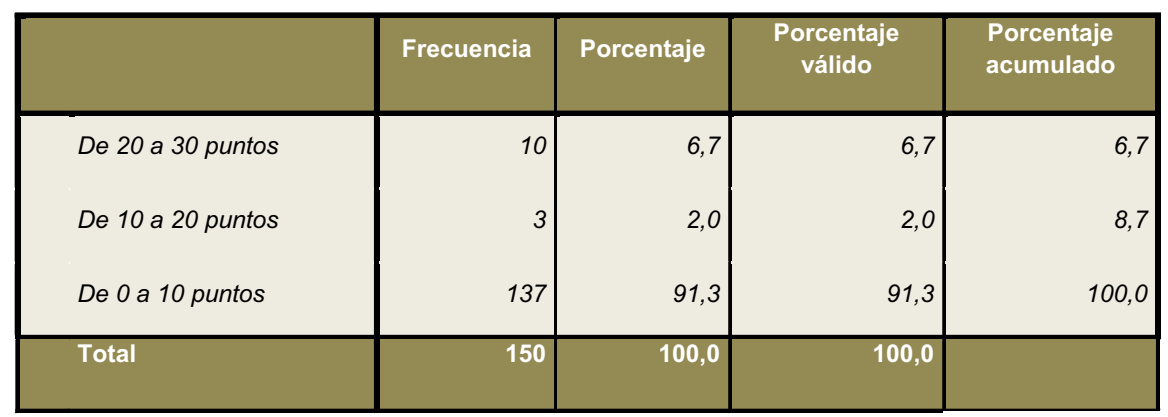

Tabla $\mathrm{N}^{\circ} 1$

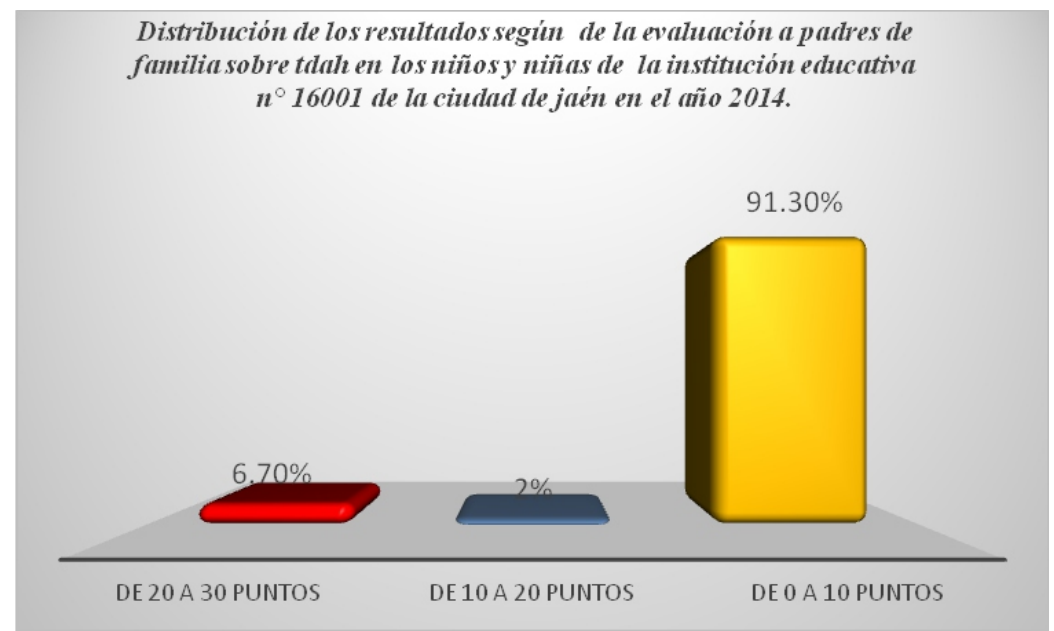

Figura $\mathrm{N}^{\circ} 1$ 
Luego de evaluar a los padres de familia de los 150 estudiantes se obtuvo que $10(6,7 \%)$ niños muestran Déficit de Atención con Hiperactividad, mientras que $3(2 \%)$ niños muestran una hiperactividad situacional y 137 (91,3\%) no presenta Déficit de Atención con Hiperactividad.

\section{RESULTADOS DE LOS LOGROS DE APRENDIZAJE EN LOS NIÑOS Y NIÑAS DEL GRUPO EXPERIMENTAL CON TDAH DE LA INSTITUCIÓN EDUCATIVA N 16001 DE LA CIUDAD DE JAÉN EN EL AÑO 2014.}

\begin{tabular}{|c|c|c|c|c|c|c|c|c|c|c|c|c|c|c|c|c|c|}
\hline \multirow{5}{*}{ AREAS } & \multicolumn{16}{|c|}{ AÑO ESCOLAR 2014} & \\
\hline & \multicolumn{16}{|c|}{ ESCALA DE CALIFICACION } & \multirow{4}{*}{ 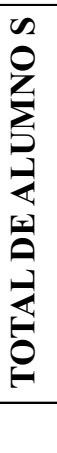 } \\
\hline & \multicolumn{8}{|c|}{ Pre test } & \multicolumn{8}{|c|}{ Post test } & \\
\hline & \multicolumn{2}{|c|}{ En Inicio } & \multicolumn{2}{|c|}{$\begin{array}{c}\text { En } \\
\text { proceso }\end{array}$} & \multicolumn{2}{|c|}{$\begin{array}{c}\text { Logro } \\
\text { previsto }\end{array}$} & \multicolumn{2}{|c|}{$\begin{array}{c}\text { Logro } \\
\text { destacado }\end{array}$} & \multicolumn{2}{|c|}{$\begin{array}{l}\text { En } \\
\text { Inicio }\end{array}$} & \multicolumn{2}{|c|}{$\begin{array}{c}\text { En } \\
\text { proceso }\end{array}$} & \multicolumn{2}{|c|}{$\begin{array}{c}\text { Logro } \\
\text { previsto }\end{array}$} & \multicolumn{2}{|c|}{$\begin{array}{c}\text { Logro } \\
\text { destacad } \\
0\end{array}$} & \\
\hline & $\mathrm{n}$ & $\%$ & $\mathrm{n}$ & $\%$ & $\mathrm{n}$ & $\%$ & $\mathrm{n}$ & $\%$ & $\mathrm{n}$ & $\%$ & $\mathrm{n}$ & $\%$ & $\mathrm{n}$ & $\%$ & $\mathrm{n}$ & $\%$ & \\
\hline $\begin{array}{l}\text { Comunicación } \\
\text { Integral }\end{array}$ & 3 & $60 \%$ & 2 & $40 \%$ & 0 & $0 \%$ & 0 & $0 \%$ & 0 & $0 \%$ & 0 & $0 \%$ & 1 & $20 \%$ & 4 & $80 \%$ & 5 \\
\hline $\begin{array}{c}\text { Lógico } \\
\text { Matemática }\end{array}$ & 3 & $60 \%$ & 2 & $40 \%$ & 0 & $0 \%$ & 0 & $0 \%$ & 0 & $0 \%$ & 0 & $0 \%$ & 1 & $20 \%$ & 4 & $80 \%$ & 5 \\
\hline $\begin{array}{c}\text { Personal } \\
\text { Social }\end{array}$ & 2 & $40 \%$ & 3 & $60 \%$ & 0 & $0 \%$ & 0 & $0 \%$ & 0 & $0 \%$ & 0 & $0 \%$ & 0 & $0 \%$ & 5 & $\begin{array}{c}100 \\
\%\end{array}$ & 5 \\
\hline $\begin{array}{l}\text { Ciencia Y } \\
\text { Ambiente }\end{array}$ & 4 & $80 \%$ & 1 & $20 \%$ & 0 & $0 \%$ & 0 & $0 \%$ & 0 & $0 \%$ & 0 & $0 \%$ & 0 & $0 \%$ & 5 & $\begin{array}{c}100 \\
\%\end{array}$ & 5 \\
\hline $\begin{array}{l}\text { Formación } \\
\text { Religiosa }\end{array}$ & 1 & $20 \%$ & 4 & $80 \%$ & 0 & $0 \%$ & 0 & $0 \%$ & 0 & $0 \%$ & 0 & $0 \%$ & 0 & $0 \%$ & 5 & $\begin{array}{c}100 \\
\%\end{array}$ & 5 \\
\hline $\begin{array}{l}\text { Educación } \\
\text { Artística }\end{array}$ & 4 & $80 \%$ & 1 & $20 \%$ & 0 & $0 \%$ & 0 & $0 \%$ & 0 & $0 \%$ & 0 & $0 \%$ & 0 & $0 \%$ & 5 & $\begin{array}{c}100 \\
\%\end{array}$ & 5 \\
\hline $\begin{array}{l}\text { Educación } \\
\text { Física }\end{array}$ & 4 & $80 \%$ & 1 & $20 \%$ & 0 & $0 \%$ & 0 & $0 \%$ & 0 & $0 \%$ & 0 & $0 \%$ & 0 & $0 \%$ & 5 & $\begin{array}{c}100 \\
\%\end{array}$ & 5 \\
\hline Ingles & 3 & $60 \%$ & 1 & $20 \%$ & 1 & $20 \%$ & 0 & $0 \%$ & 0 & $0 \%$ & 0 & $0 \%$ & 0 & $0 \%$ & 5 & $\begin{array}{c}100 \\
\%\end{array}$ & 5 \\
\hline Computación & 2 & $40 \%$ & 3 & $60 \%$ & 0 & $0 \%$ & 0 & $0 \%$ & 0 & $0 \%$ & 0 & $0 \%$ & 0 & $0 \%$ & 5 & $\begin{array}{c}100 \\
\%\end{array}$ & 5 \\
\hline
\end{tabular}

Tabla $\mathrm{N}^{\circ} 2$

Fuente: Evaluación aplicada a los alumnos y alumnas de tercer grado de educación primaria de la Institución Educativa "Ramón Castilla y Marquesado" Nº 16001 de Jaén- 2014 


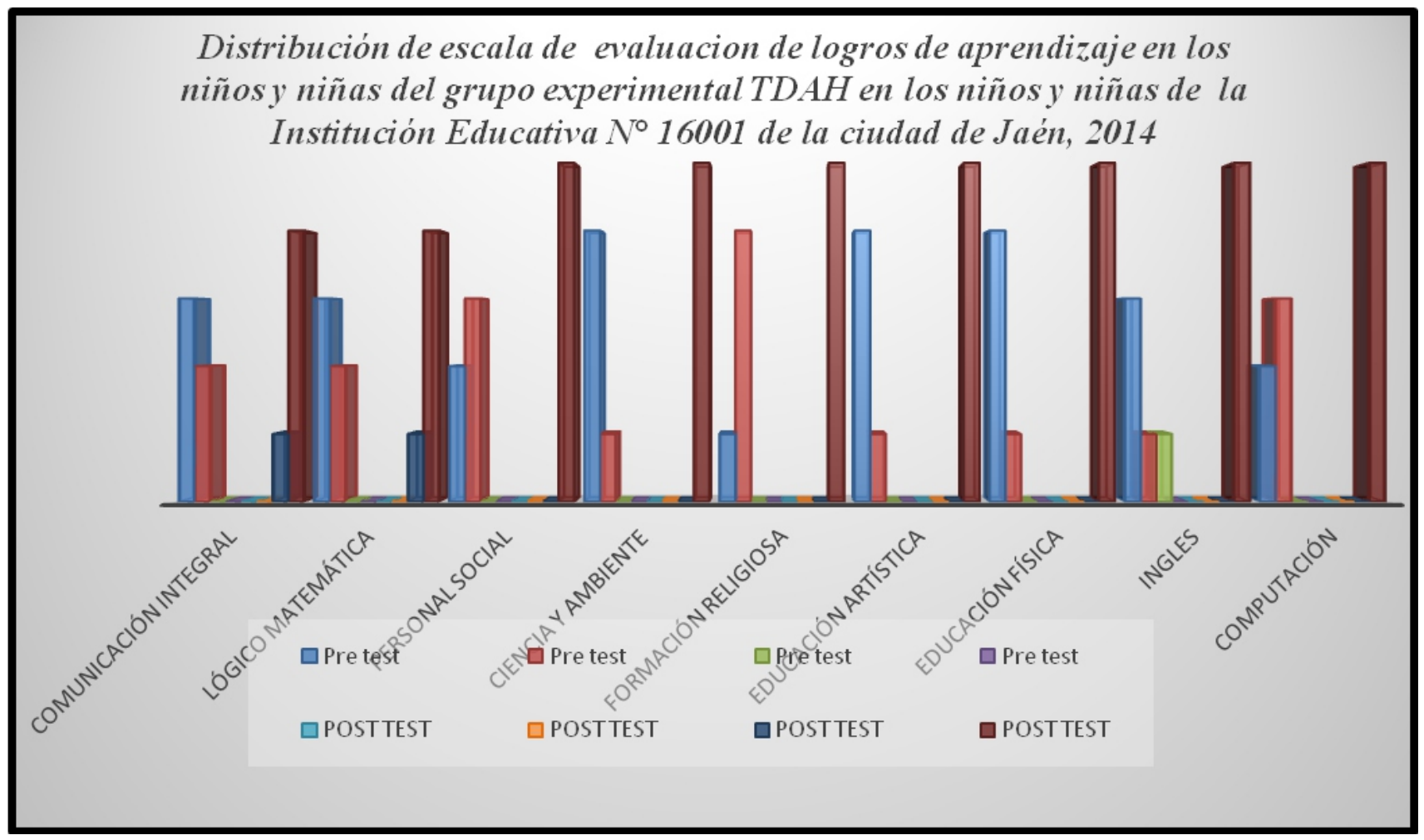

Tabla $\mathrm{N}^{\circ} 3$

\section{Descripción}

En la tabla se muestra los resultados de la evaluación del grupo control como sigue:

En el pre test se observa que el curso de comunicación integral 3(60\%) de los estudiantes está con un nivel de aprendizaje en inicio, y 2(40\%) de los estudiantes están con un nivel de aprendizaje en proceso. en la asignatura de matemática se tiene que $3(60 \%)$ de los estudiantes están en inicio, y 2(40\%) de los estudiantes están en proceso; en Personal Social, el nivel de aprendizaje es 2(40\%) están en inicio, y $3(60 \%)$ de los estudiantes están en proceso; en el área de ciencia y ambiente los niveles de aprendizaje que presentaron los estudiantes es $4(80 \%)$ están en inicio, 1(20\%) está en proceso; en formación religiosa el nivel de aprendizaje es $1(20 \%)$ están en inicio, y 4(80\%) de los estudiantes están en proceso; en cuanto al nivel de aprendizaje en la asignatura de Educación artística se tiene que 4(80\%) de los estudiantes están en inicio y $1(20 \%)$ están en proceso; en Educación física el nivel de aprendizaje es 4(80\%) están en inicio, y 1(20\%) están en proceso; en idioma Inglés los niveles de aprendizaje que presentaron los estudiantes es $3(60 \%)$ están en inicio, $1(20 \%)$ está en proceso y $1(20 \%)$ está en logro previsto; en computación el nivel de aprendizaje es 2(40\%) están en inicio, y 3(60\%) de los estudiantes están en proceso.

En el post test luego de aplicado el programa se obtuvo en el curso de comunicación integral 1(20\%) de los estudiantes está con un nivel de aprendizaje de logro previsto y 4(80\%) de los estudiantes están con un nivel de logro destacado; en la asignatura de matemática se tiene que 1(20\%) de los estudiantes está con un nivel de aprendizaje de logro previsto y 4(80\%) están con un nivel de logro destacado; en personal social el nivel de aprendizaje es $5(100 \%)$ están con un nivel de logro destacado; en ciencia y ambiente los niveles de aprendizaje que presentaron los estudiantes es 5(100\%) están con un nivel de logro destacado; en formación religiosa el nivel de aprendizaje es 5(100\%) de los estudiantes están con un nivel de logro destacado; en educación artística se tiene 5(100\%) están con un nivel destacado; 
en educación física el nivel de aprendizaje es $5(100 \%)$ de los estudiantes están con un nivel de logro destacado; en Inglés los niveles de aprendizaje que presentaron los estudiantes $5(100 \%)$, nivel destacado y en la asignatura de computación el nivel de aprendizaje es 5(100\%) están con un nivel de logro destacado.

\section{DISCUSIÓN}

En relación al diagnóstico del TDAH los resultados arrojaron que 10 estudiantes presentan signos y síntomas de TDHA.

El logro de aprendizaje de los niños y niñas con TDAH en la IE. $N^{\circ} 16001$ de la ciudad de Jaén en el año 2014, antes de la aplicación del programa, su nivel está en inicio; en consecuencia, los problemas escolares más frecuentemente relatados por padres y profesores de un niño o adolescente con TDAH, conllevan un mayor riesgo de repetir la asignatura, porque los estudiantes no presentan sus actividades académicas, poseen escasa comprensión del material trabajado, malos hábitos de estudio, poca participación en clase, no piden ayuda al profesor/a, notas por debajo de su potencial, suspensos, conducta disruptiva en clase, enfrentamientos.

Se diseñó y aplico el programa de estrategias, para mejorar los logros de aprendizaje en los niños y niñas con TDAH de la institución educativa $\mathrm{N}^{\circ} 16001$, basándose en las teorías de asimilación y acomodación de Jean Piaget.

Los logros de aprendizaje de los niños y niñas con TDAH en la IE. $N^{\circ} 16001$ de la ciudad de Jaén en el año 2014, después de la aplicación del programa es destacado, esto se corrobora con los resultados hallados por Lefa Eddy Ives (2010). En demasiadas ocasiones, los padres y profesores interpretan que el hijo o alumno no se esfuerza, no trabaja, ni tiene interés por el estudio, pero, cuando uno profundiza en el problema, constata que sí hay interés y ganas de hacer las cosas bien, pero existe una disfunción a nivel de las funciones ejecutivas que hace necesario que se le guíe y controle de cerca para mejorar las técnicas de estudio, la capacidad de planificar y de organizarse, pudiendo así lograr, en la mayoría de ocasiones, una mejoría en el rendimiento académico.

\section{CONCLUSIONES.}

- Se diagnosticaron a través de la Escala de Conners 10 casos de estudiantes con TDH con déficit de atención, utilizándose para este estudio 5 niños para el grupo experimental y 5 niños para el grupo control.

- Los logros de aprendizaje de los niños del grupo experimental y control antes del programa están catalogados según el DCN, 2009 como en inicio.

- Se diseñó y aplico el Programa de Estrategias y fueron validados por dos expertos y aplicado al grupo experimental.

- Los logros de aprendizaje de los niños del grupo experimental después del programa están considerados como logros destacados.

\section{AGRADECIMIENTO.}

Nuestro especial agradecimiento a la Universidad Nacional de Jaén, por brindar espacios para la divulgación y difusión del conocimiento.

Asimismo, nuestro agradecimiento a la IE. N 16001 de la ciudad de Jaén, por las facilidades brindadas durante todo el proceso de la ejecución de la investigación, así como a los profesionales que colaboraron con sus valiosos aportes y sugerencias.

\section{REFERENCIAS BIBLIOGRÁFICAS.}

Alfonso, I. (2003). Elementos conceptuales básicos del proceso de enseñanza-aprendizaje. Disponible en http://www.scielo.sld.cu/scielo. php. 
Castañeda, C. (2005). Los problemas sociales en el trastorno por déficit de atención con hiperactividad (TDAH).

Díaz-Barriga, F. y Hernández, G. (2002). Estrategias docentes para un aprendizaje significativo, una interpretación constructivista.

México: Mc Graw Hill.

Duarte, J. (2004). Inadaptación infantil y su relación con el rendimiento académico. Tesis de Maestría en Ciencias de la Educación, Universidad del Mayab, Yucatán, México.

García, H. y Ugarte, D. (1997) Resolviendo conflictos en la escuela. Manual para maestros. Lima:APENAC.

Gardner, H. (1995). Inteligencias múltiples. Barcelona: Paidós.

Gardner, H. (1997). Estructura de la mente. La teoría de las múltiples inteligencias. México: Fondo de Cultura Económica.

Girard, K. y Koch, S. (1997). Resolución de conflictos en las escuelas. Argentina: Granica.

Godenzzi, J. (2001). Pedagogía del encuentro. El sujeto, la convivencia y el conocimiento. Lima: programa Forte.

Goleman, D. (1998). Inteligencia emocional. Barcelona: Cairos.

Rangel Araiza, José Francisco. (2014). El trastorno por déficit de atención con y sin hiperactividad (TDA/H) y la violencia: Revisión de la bibliografía. Salud mental, 37(1).

\section{CORRESPONDENCIA}

Nazario Aguirre Baique.

Villanueva Pinillos $N^{\circ} 720$ - Jaén - Cajamarca

Teléf. 943487530

nabunj@hotmail.com

A

